\title{
Some Results on Preconditioned Mixed-Type Splitting Iterative Method
}

\author{
Guangbin Wang ${ }^{1}$ and Fuping $\operatorname{Tan}^{2}$ \\ ${ }^{1}$ Department of Mathematics, Qingdao University of Science and Technology, Qingdao 266061, China \\ ${ }^{2}$ Department of Mathematics, Shanghai University, Shanghai 200444, China
}

Correspondence should be addressed to Guangbin Wang; wguangbin750828@sina.com

Received 20 June 2013; Revised 14 September 2013; Accepted 27 September 2013

Academic Editor: Zhong-Zhi Bai

Copyright (C) 2013 G. Wang and F. Tan. This is an open access article distributed under the Creative Commons Attribution License, which permits unrestricted use, distribution, and reproduction in any medium, provided the original work is properly cited.

We present a preconditioned mixed-type splitting iterative method for solving the linear system $A x=b$, where $A$ is a Z-matrix. And we give some comparison theorems to show that the rate of convergence of the preconditioned mixed-type splitting iterative method is faster than that of the mixed-type splitting iterative method. Finally, we give one numerical example to illustrate our results.

\section{Introduction}

For solving linear system,

$$
A x=b,
$$

where $A$ is an $n \times n$ square matrix and $x$ and $b$ are $n$-dimensional vectors, the basic iterative method is

$$
M x^{k+1}=N x^{k}+b, \quad k=0,1, \ldots,
$$

where $A=M-N$ and $M$ is nonsingular. Thus, (2) can be written as

$$
x^{k+1}=T x^{k}+c, \quad k=0,1, \ldots,
$$

where $T=M^{-1} N$ and $c=M^{-1} b$.

Assuming that $A$ has unit diagonal entries, let $A=I-L-$ $U$, where $I$ is the identity matrix and $-L$ and $-U$ are strictly lower and strictly upper triangular parts of $A$, respectively.

Transform the original system (1) into the preconditioned form as follows:

$$
P A x=P b \text {. }
$$

Then, we can define the basic iterative scheme as follows:

$$
M_{p} x^{k+1}=N_{p} x^{k}+P b, \quad k=0,1, \ldots,
$$

where $P A=M_{p}-N_{p}$ and $M_{p}$ is nonsingular. Thus, the equation above can also be written as

$$
x^{k+1}=T x^{k}+c, \quad k=0,1, \ldots,
$$

where $T=M_{p}^{-1} N_{p}$ and $c=M_{p}^{-1} P b$.

In paper [1], Cheng et al. presented the mixed-type splitting iterative method as follows:

$$
\begin{array}{r}
\left(D+D_{1}+L_{1}-L\right) x^{k+1}=\left(D_{1}+L_{1}+U\right) x^{k}+b, \\
k=0,1,2 \ldots,
\end{array}
$$

with the following iterative matrix:

$$
T=\left(D+D_{1}+L_{1}-L\right)^{-1}\left(D_{1}+L_{1}+U\right),
$$

where $D_{1}$ is an auxiliary nonnegative diagonal matrix, $L_{1}$ is an auxiliary strictly lower triangular matrix, and $0 \leq L_{1} \leq L$.

In this paper, we will establish the preconditioned mixedtype splitting iterative method with the preconditioners $P_{\alpha}=$ $I+\alpha L, P_{\beta}=I+\beta U$, and $P_{\alpha \beta}=I+\alpha L+\beta U$ for solving linear systems. And we obtain some comparison results which show that the rate of convergence of the preconditioned mixed-type splitting iterative method with $P_{\alpha \beta}$ is faster than that of the preconditioned mixed-type splitting iterative method with $P_{\alpha}$ or $P_{\beta}$. Finally, we give one numerical example to illustrate our results. 


\section{Preconditioned Mixed-Type Splitting Iterative Method}

For the linear system (1), we consider its preconditioned form as follows:

$$
P_{\alpha \beta} A x=P_{\alpha \beta} b,
$$

with the preconditioner $P_{\alpha \beta}=I+\alpha L+\beta U$; that is,

$$
A_{\alpha \beta} x=b_{\alpha \beta}
$$

We apply the mixed-type splitting iterative method to it and have the corresponding preconditioned mixed-type splitting iterative method as follows:

$$
\begin{array}{r}
\left(D_{\alpha \beta}+D_{1}+L_{1}-L_{\alpha \beta}\right) x^{k+1}=\left(D_{1}+L_{1}+U_{\alpha \beta}\right) x^{k}+b_{\alpha \beta}, \\
k=0,1,2, \ldots,
\end{array}
$$

that is,

$$
\begin{aligned}
x^{k+1}= & \left(D_{\alpha \beta}+D_{1}+L_{1}-L_{\alpha \beta}\right)^{-1}\left(D_{1}+L_{1}+U_{\alpha \beta}\right) x^{k} \\
& +\left(D_{\alpha \beta}+D_{1}+L_{1}-L_{\alpha \beta}\right)^{-1} b_{\alpha \beta}, \quad k=0,1,2 \cdots,
\end{aligned}
$$

So, the iterative matrix is

$$
\widetilde{T}=\left(D_{\alpha \beta}+D_{1}+L_{1}-L_{\alpha \beta}\right)^{-1}\left(D_{1}+L_{1}+U_{\alpha \beta}\right),
$$

where $D_{\alpha \beta},-L_{\alpha \beta}$, and $-U_{\alpha \beta}$ are the diagonal, strictly lower, and strictly upper triangular matrices obtained from $A_{\alpha \beta}, D_{1}$ is an auxiliary nonnegative diagonal matrix, $L_{1}$ is an auxiliary strictly lower triangular matrix, and $0 \leq L_{1} \leq L_{\alpha \beta}$.

If we choose $\beta=0$, we have the following corresponding iterative matrix:

$$
\bar{T}=\left(D_{\alpha}+D_{1}+L_{1}-L_{\alpha}\right)^{-1}\left(D_{1}+L_{1}+U_{\alpha}\right) .
$$

And if we choose $\alpha=0$, we have the following corresponding iterative matrix:

$$
\widehat{T}=\left(D_{\beta}+D_{1}+L_{1}-L_{\beta}\right)^{-1}\left(D_{1}+L_{1}+U_{\beta}\right) .
$$

If we choose certain auxiliary matrices, we can get the classical iterative methods as follows.

(1) The PSOR method is

$$
\begin{gathered}
D_{1}=\frac{1}{r}(1-r) D_{\alpha \beta}, \quad L_{1}=0, \\
\widetilde{L}_{r}=\left(D_{\alpha \beta}-r L_{\alpha \beta}\right)^{-1}\left[(1-r) D_{\alpha \beta}+r U_{\alpha \beta}\right] .
\end{gathered}
$$

(2) The PAOR method is

$$
\begin{gathered}
D_{1}=\frac{1}{w}(1-w) D_{\alpha \beta}, \quad L_{1}=\frac{1}{w}(w-r) L_{\alpha \beta}, \\
\widetilde{L}_{r w}=\left(D_{\alpha \beta}-r L_{\alpha \beta}\right)^{-1}\left[(1-w) D_{\alpha \beta}+(w-r) L_{\alpha \beta}+w U_{\alpha \beta}\right] .
\end{gathered}
$$

We need the following definitions and results.

Definition 1 (see [2]). A matrix $A$ is a $Z$-matrix if $a_{i j} \leq 0$, for all $i, j=1,2, \ldots n$, such that $i \neq j$. A matrix $A$ is an $L$-matrix if $a_{i i}>0, i=1,2, \ldots n$, and $a_{i j} \leq 0$, for all $i, j=1,2, \ldots n$, such that $i \neq j$.

Definition 2 (see [2]). A matrix $A$ is an $M$-matrix if $A$ is a nonsingular $Z$-matrix, and $A^{-1} \geq 0$.

Definition 3 (see $[2,3]$ ). Let $M, N \in R^{n, n}$. Then, $A=M-N$ is called a regular splitting if $M^{-1} \geq 0$ and $N \geq 0 ; A=M-N$ is called an $M$-splitting if $M$ is an $M$-matrix, $N \geq 0$.

Lemma 4 (see [2]). Let $A \geq 0$ be an irreducible matrix. Then,

(1) A has a positive real eigenvalue equal to its spectral radius;

(2) to $\rho(A)$, there corresponds an eigenvector $x>0$;

(3) $\rho(A)$ is a simple eigenvalue of $A$.

Lemma 5 (see [4]). Let $A$ be a nonnegative matrix. Then,

(1) if $\alpha x \leq A x$ for some nonnegative vector $x, x \neq 0$, then $\alpha \leq \rho(A)$

(2) if $A x \leq \beta x$ for some positive vector $x$, then $\rho(A) \leq \beta$. Moreover, if $A$ is irreducible and if $0 \neq \alpha x \leq A x \leq \beta x$ for some nonnegative vectors $x$, then

$$
\alpha \leq \rho(A) \leq \beta .
$$

Lemma 6 (see [5]). Let $A=M-N$ be an $M$-splitting of $A$. Then, $\rho\left(M^{-1} N\right)<1$ if and only if $A$ is a nonsingular $M$ matrix.

Lemma 7 (see $[6,7])$. Let $A$ be a Z-matrix. Then, $A$ is a nonsingular $M$-matrix if and only if there is a positive vector $x$ such that $A x \geq 0$.

Lemma 8 (see [8]). Let $A=M-N$ be a regular splitting of $A$. Then, the splitting is convergent if and only if $A^{-1} \geq 0$.

Lemma 9 (see [9]). Let $A$ and $B$ be two $n \times n$ nonsingular lower triangular L-matrices. If $A \geq B$, then $B^{-1} \geq A^{-1} \geq 0$.

\section{Convergence Analysis and Comparison Results}

Theorem 10. Let $A$ be a nonsingular Z-matrix. Assume that $D_{1} \geq 0,0 \leq L_{1} \leq L_{\alpha}, \alpha \in[0,1]$, and $\bar{T}$ and $T$ are the iterative 
matrices given by (14) and (8), respectively. Consider the following.

(i) If $\rho(T)<1$, then $\rho(\bar{T})<\rho(T)<1$.

(ii) Let $A$ be irreducible. Assume that $1-\alpha \sum_{l=1}^{i-1} a_{i l} a_{l j}>0$ and $a_{i j}+\sum_{k=i+1}^{n} a_{i k} a_{k j} \leq 0$;

then, one has

(1) $\rho(\bar{T}) \geq \rho(T)$, if $\rho(T) \geq 1$,

(2) $\rho(\bar{T}) \leq \rho(T)$, if $\rho(T)<1$.

Proof. Let

$$
\begin{gathered}
M_{\alpha}=D_{\alpha}+D_{1}+L_{1}-L_{\alpha}, \\
N_{\alpha}=D_{1}+L_{1}+U_{\alpha}, \\
M=I+D_{1}+L_{1}-L, \\
N=D_{1}+L_{1}+U, \\
E_{\alpha}=(I+\alpha L)\left(I+D_{1}+L_{1}-L\right), \\
F_{\alpha}=(I+\alpha L)\left(D_{1}+L_{1}+U\right) .
\end{gathered}
$$

Then, we have

$$
A=M-N, \quad A_{\alpha}=M_{\alpha}-N_{\alpha}=E_{\alpha}-F_{\alpha} .
$$

(i) Since $A$ is a nonsingular Z-matrix and $D_{1} \geq 0,0 \leq L_{1} \leq$ $L_{\alpha}$, it is clear that $M=I+D_{1}+L_{1}-L$ is a nonsingular $M$ matrix and the splitting

$$
A=M-N=\left(I+D_{1}+L_{1}-L\right)-\left(D_{1}+L_{1}+U\right)
$$

is an $M$-splitting. Since $\rho(T)<1$, it follows from Lemma 6 that $A$ is a nonsingular $M$-matrix. Then, by Lemma 7 , there is a positive vector $x$ such that $A x \geq 0$, so $A_{\alpha} x=(I+\alpha L) A x \geq 0$.

By Lemma $7, A_{\alpha}$ is also a nonsingular $M$-matrix.

Obviously, we can get that $D_{\alpha}$ is a positive diagonal matrix. And from $L_{\alpha}$ is nonnegative, we know that $M_{\alpha}$ being a $Z$-matrix. Since $D_{\alpha}^{-1} L_{\alpha} \geq 0$ is a strictly lower triangular matrix, so that $\rho\left(D_{\alpha}^{-1} L_{\alpha}\right)=0<1$.

So, we have $\left(I+D_{\alpha}^{-1} D_{1}+D_{\alpha}^{-1} L_{1}-D_{\alpha}^{-1} L_{\alpha}\right)^{-1} \geq 0$.

Then, $M_{\alpha}^{-1}=\left(I+D_{\alpha}^{-1} D_{1}+D_{\alpha}^{-1} L_{1}-D_{\alpha}^{-1} L_{\alpha}\right)^{-1} D_{\alpha}^{-1} \geq 0$; hence, $M_{\alpha}$ is a nonsingular $M$-matrix.

For $j \neq i+1$, it is obvious that $\left(U_{\alpha}\right)_{i j}=-a_{i j}+\alpha a_{i i+1} a_{i+1 j} \geq 0$. And for $j=i+1$, we have $\left(U_{\alpha}\right)_{i j}=(\alpha-1) a_{i j} \geq 0$. Thus, $U_{\alpha} \geq 0$ and $N_{\alpha} \geq 0$.

We have proven that $A_{\alpha}=M_{\alpha}-N_{\alpha}$ and $A=M-N$ are both $M$-splittings and $E_{\alpha}^{-1} F_{\alpha}=M^{-1} N$, two splittings $A_{\alpha}=$ $M_{\alpha}-N_{\alpha}=E_{\alpha}-F_{\alpha}$ are nonnegative.
On the other hand, since $L_{\alpha}=D_{\alpha}-I+\alpha \bar{D}+L-\alpha L+$ $\alpha L^{2}+\alpha \bar{E}$, we get

$$
\begin{aligned}
E_{\alpha}-M_{\alpha}= & (I+\alpha L)\left(I+D_{1}+L_{1}-L\right) \\
& -\left(D_{\alpha}+D_{1}+L_{1}-L_{\alpha}\right) \\
= & \left(I+D_{1}+L_{1}-L\right)+\alpha L\left(I+D_{1}+L_{1}-L\right) \\
& -\left(D_{\alpha}+D_{1}+L_{1}-L_{\alpha}\right) \\
= & I-L+\alpha L+\alpha L D_{1}+\alpha L L_{1}-\alpha L^{2}-D_{\alpha}+L_{\alpha} \\
= & I-L+\alpha L+\alpha L D_{1}+\alpha L L_{1}-\alpha L^{2}-D_{\alpha}+D_{\alpha} \\
& -I+\alpha \bar{D}+L-\alpha L+\alpha L^{2}+\alpha \bar{E} \\
= & \alpha\left(L D_{1}+L L_{1}+\bar{D}+\bar{E}\right) \geq 0,
\end{aligned}
$$

which implies that

$$
A_{\alpha}^{-1} E_{\alpha}-A_{\alpha}^{-1} M_{\alpha}=A_{\alpha}^{-1}\left(E_{\alpha}-M_{\alpha}\right) \geq 0 .
$$

Therefore, $A_{\alpha}^{-1} E_{\alpha} \geq A_{\alpha}^{-1} M_{\alpha} \geq 0$. So, we have $\rho\left(M_{\alpha}^{-1} N_{\alpha}\right) \leq$ $\rho\left(E_{\alpha}^{-1} F_{\alpha}\right)$; that is,

$$
\rho(\bar{T}) \leq \rho(T)<1
$$

(ii) Let $A=I-L-U$ be irreducible. Since $L+U$ is a nonnegative and irreducible matrix, and according to the proof of Lemma 4 in paper [9], we can obtain that $\bar{T}$ and $T$ are nonnegative and irreducible matrices. Thus, from Lemma 4, we know that there exists a positive vector $x=\left(x_{1}, x_{2} \cdots x_{n}\right)^{T}$ such that $T x=\lambda x$, where we denote $\lambda=\rho(T)$, which is equivalent to

$$
\begin{gathered}
\left(D_{1}+L_{1}+U\right) x=\lambda\left(I+D_{1}+L_{1}-L\right) x, \\
(U-\lambda I+\lambda L) x=\left[(\lambda-1) D_{1}+(\lambda-1) L_{1}\right] x .
\end{gathered}
$$

Let $L U=\bar{D}+\bar{E}+\bar{F}$, where $\bar{D}, \bar{E}$, and $\bar{F}$ are the diagonal, lower triangular, and upper triangular parts of $L U$, respectively. So,

$$
\begin{aligned}
A_{\alpha} & =D_{\alpha}-L_{\alpha}-U_{\alpha} \\
& =(I-\alpha \bar{D})-\left(L-\alpha L+\alpha L^{2}+\alpha \bar{E}\right)-(U+\alpha \bar{F}),
\end{aligned}
$$

where $D_{\alpha}=I-\alpha \bar{D}, L_{\alpha}=L-\alpha L+\alpha L^{2}+\alpha \bar{E}, U_{\alpha}=U+\alpha \bar{F}$.

Now, we consider

$$
\begin{aligned}
\bar{T} x-T x= & \left(D_{\alpha}+D_{1}+L_{1}-L_{\alpha}\right)^{-1} \\
& \times\left(D_{1}+L_{1}+U_{\alpha}\right) x-\lambda x \\
= & \left(D_{\alpha}+D_{1}+L_{1}-L_{\alpha}\right)^{-1} \\
& \times\left[\left(D_{1}+L_{1}+U_{\alpha}\right)-\lambda\left(D_{\alpha}+D_{1}+L_{1}-L_{\alpha}\right)\right] x \\
= & \left(D_{\alpha}+D_{1}+L_{1}-L_{\alpha}\right)^{-1}
\end{aligned}
$$




$$
\begin{aligned}
& \times\left[\left(D_{1}+L_{1}+U+\alpha \bar{F}\right)\right. \\
& -\lambda\left(I-\alpha \bar{D}+D_{1}+L_{1}\right. \\
& \left.\left.-L+\alpha L-\alpha L^{2}-\alpha \bar{E}\right)\right] x \\
= & \left(D_{\alpha}+D_{1}+L_{1}-L_{\alpha}\right)^{-1} \\
& \times\left\{\left[\left(D_{1}+L_{1}+U\right)-\lambda\left(I+D_{1}+L_{1}-L\right)\right] x\right. \\
& \left.+\left[\alpha \bar{F}+\lambda\left(\alpha \bar{D}-\alpha L+\alpha L^{2}+\alpha \bar{E}\right)\right] x\right\} \\
= & \left(D_{\alpha}+D_{1}+L_{1}-L_{\alpha}\right)^{-1} \\
& \times\left[\alpha \bar{F}+\lambda\left(\alpha \bar{D}-\alpha L+\alpha L^{2}+\alpha \bar{E}\right)\right] x \\
= & \left(D_{\alpha}+D_{1}+L_{1}-L_{\alpha}\right)^{-1} \\
& \times\left[(\lambda-1) \alpha L\left(D_{1}+L_{1}\right)+(\lambda-1) \alpha(\bar{D}+\bar{E})\right] x \\
= & (\lambda-1)\left(D_{\alpha}+D_{1}+L_{1}-L_{\alpha}\right)^{-1} \\
& \times \alpha\left[L D_{1}+L L_{1}+\bar{D}+\bar{E}\right] x .
\end{aligned}
$$

Since $D_{\alpha}+D_{1}+L_{1}-L_{\alpha}$ is an $M$-matrix, and $L D_{1}+L L_{1}+\bar{D}+\bar{E} \geq$ 0 , we have the following.

(1) If $\lambda \geq 1$, then $\bar{T} x \geq T x=\lambda x$. By Lemma 5, we get $\rho(\bar{T}) \geq \rho(T)$.

(2) If $\lambda<1$, then $\bar{T} x \leq T x=\lambda x$. By Lemma 5 , we get $\rho(\bar{T}) \leq \rho(T)$.

Theorem 11. Let $A$ be a nonsingular Z-matrix. Assume that $D_{1} \geq 0,0 \leq L_{1} \leq L_{\alpha \beta}, \alpha, \beta \in[0,1]$, and $\widetilde{T}$ and $T$ are the iterative matrices given by (13) and (8), respectively. Consider the following.

(i) If $\rho(T)<1$, then

$$
\rho(\widetilde{T})<\rho(T)<1
$$

(ii) Let $A$ be irreducible. Assume that

$$
\begin{gathered}
1-\alpha \sum_{l=1}^{i-1} a_{i l} a_{l j}-\beta \sum_{k=i+1}^{n} a_{i k} a_{k j}>0, \\
a_{i j}+\sum_{k=i+1}^{n} a_{i k} a_{k j} \leq 0
\end{gathered}
$$

Then, one has

(1) $\rho(\widetilde{T}) \geq \rho(T)$ if $\rho(T) \geq 1$,
(2) $\rho(\widetilde{T}) \leq \rho(T)$ if $\rho(T)<1$.
Proof. Let

$$
\begin{gathered}
M_{\alpha \beta}=D_{\alpha \beta}+D_{1}+L_{1}-L_{\alpha \beta}, \\
N_{\alpha \beta}=D_{1}+L_{1}+U_{\alpha \beta}, \\
M=I+D_{1}+L_{1}-L, \\
N=D_{1}+L_{1}+U, \\
E_{\alpha}=(I+\alpha L+\beta U)\left(I+D_{1}+L_{1}-L\right), \\
F_{\alpha}=(I+\alpha L+\beta U)\left(D_{1}+L_{1}+U\right) .
\end{gathered}
$$

Then, we have

$$
A=M-N, \quad A_{\alpha \beta}=M_{\alpha \beta}-N_{\alpha \beta}=E_{\alpha \beta}-F_{\alpha \beta} .
$$

(i) By a similar proof of Theorem 10, we can prove that $A_{\alpha \beta}=M_{\alpha \beta}-N_{\alpha \beta}$ and $A=M-N$ are both $M$-splitting and $E_{\alpha \beta}^{-1} F_{\alpha \beta}=M^{-1} N$, two splittings $A_{\alpha \beta}=M_{\alpha \beta}-N_{\alpha \beta}=E_{\alpha \beta}-F_{\alpha \beta}$, are nonnegative.

On the other hand, since $L_{\alpha \beta}=D_{\alpha \beta}-I+\alpha \bar{D}+\beta \widehat{D}+L-$ $\alpha L+\alpha L^{2}+\alpha \bar{E}+\beta \widehat{E}$, we get

$$
\begin{aligned}
E_{\alpha \beta}-M_{\alpha \beta}= & (I+\alpha L+\beta U)\left(I+D_{1}+L_{1}-L\right) \\
& -\left(D_{\alpha \beta}+D_{1}+L_{1}-L_{\alpha \beta}\right) \\
= & \left(I+D_{1}+L_{1}-L\right)+\alpha L\left(I+D_{1}+L_{1}-L\right) \\
& +\beta U\left(I+D_{1}+L_{1}-L\right) \\
& -\left(D_{\alpha \beta}+D_{1}+L_{1}-L_{\alpha \beta}\right) \\
= & I-L+\alpha L\left(I+D_{1}+L_{1}-L\right) \\
& +\beta U\left(I+D_{1}+L_{1}-L\right)-D_{\alpha \beta}+L_{\alpha \beta} \\
= & I-L+\alpha L\left(I+D_{1}+L_{1}-L\right) \\
& +\beta U\left(I+D_{1}+L_{1}-L\right)-D_{\alpha \beta}+D_{\alpha \beta} \\
& -I+\alpha \bar{D}+\beta \widehat{D}+L-\alpha L \\
& +\alpha L^{2}+\alpha \bar{E}+\beta \widehat{E} \\
= & \beta(U-\widehat{F})+\beta U\left(D_{1}+L_{1}\right) \\
& +\alpha(\bar{D}+\bar{E})+\alpha L\left(D_{1}+L_{1}\right) \geq 0, \\
& (U)+L)
\end{aligned}
$$

which implies that

$$
A_{\alpha \beta}^{-1} E_{\alpha \beta}-A_{\alpha \beta}^{-1} M_{\alpha \beta}=A_{\alpha \beta}^{-1}\left(E_{\alpha \beta}-M_{\alpha \beta}\right) \geq 0 .
$$

Therefore, $A_{\alpha \beta}^{-1} E_{\alpha \beta} \geq A_{\alpha \beta}^{-1} M_{\alpha \beta} \geq 0$. So, we have $\rho\left(M_{\alpha \beta}^{-1} N_{\alpha \beta}\right) \leq \rho\left(E_{\alpha \beta}^{-1} F_{\alpha \beta}\right)$; that is,

$$
\rho(\bar{T}) \leq \rho(T)<1 .
$$


(ii) Let $A_{\alpha \beta}=P_{\alpha \beta} A=(I+\alpha L+\beta U) A=I-L-U+\alpha L(I-$ $L-U)+\beta U(I-L-U)$

$$
\begin{aligned}
= & I-\alpha \bar{D}-\beta \widehat{D}-\left(L-\alpha L+\alpha L^{2}+\alpha \bar{E}+\beta \widehat{E}\right) \\
& -\left(U+\alpha \bar{F}-\beta U+\beta \widehat{F}+\beta U^{2}\right),
\end{aligned}
$$

where $L U=\bar{D}+\bar{E}+\bar{F}, U L=\widehat{D}+\widehat{E}+\widehat{F}$, and $\bar{D}, \bar{E}, \bar{F}, \widehat{D}, \widehat{E}$, and $\widehat{F}$ are the diagonal, strictly lower, and strictly upper triangular matrices of $L U$ and $U L$, respectively.

(35), we have

And denote $A_{\alpha \beta}=D_{\alpha \beta}-L_{\alpha \beta}-U_{\alpha \beta}$; then according to

$$
\begin{gathered}
D_{\alpha \beta}=I-\alpha \bar{D}-\beta \widehat{D}, \\
L_{\alpha}=L-\alpha L+\alpha L^{2}+\alpha \bar{E}+\beta \widehat{E}, \\
U_{\alpha \beta}=U-\beta U+\beta U^{2}+\alpha \bar{F}+\beta \widehat{F} .
\end{gathered}
$$

By (25), we have

$$
\begin{aligned}
& \widetilde{T} x-\lambda x=\left(D_{\alpha \beta}+D_{1}+L_{1}-L_{\alpha \beta}\right)^{-1} \\
& \times\left(D_{1}+L_{1}+U_{\alpha \beta}\right) x-\lambda x \\
& =\left(D_{\alpha \beta}+D_{1}+L_{1}-L_{\alpha \beta}\right)^{-1} \\
& \times\left[\left(D_{1}+L_{1}+U_{\alpha \beta}\right)\right. \\
& \left.-\lambda\left(D_{\alpha \beta}+D_{1}+L_{1}-L_{\alpha \beta}\right)\right] x \\
& =\left(D_{\alpha \beta}+D_{1}+L_{1}-L_{\alpha \beta}\right)^{-1} \\
& \times\left[\left(D_{1}+L_{1}+U+\alpha \bar{F}-\beta U+\beta \widehat{F}+\beta U^{2}\right)\right. \\
& -\lambda\left(I-\alpha \bar{D}-\beta \widehat{D}+D_{1}+L_{1}-L\right. \\
& \left.\left.+\alpha L-\alpha L^{2}-\alpha \bar{E}-\beta \widehat{E}\right)\right] x \\
& =\left(D_{\alpha \beta}+D_{1}+L_{1}-L_{\alpha \beta}\right)^{-1} \\
& \times\left\{\left[\left(D_{1}+L_{1}+U\right)-\lambda\left(I+D_{1}+L_{1}-L\right)\right] x\right. \\
& +\left[\alpha \bar{F}-\beta U+\beta \widehat{F}+\beta U^{2}\right. \\
& -\lambda(-\alpha \bar{D}-\beta \widehat{D}+\alpha L \\
& \left.\left.\left.-\alpha L^{2}-\alpha \bar{E}-\alpha \widehat{E}\right)\right] x\right\} \\
& =\left(D_{\alpha \beta}+D_{1}+L_{1}-L_{\alpha \beta}\right)^{-1} \\
& \times\left[\alpha \bar{F}-\beta U+\beta \widehat{F}+\beta U^{2}\right. \\
& \left.-\lambda\left(-\alpha \bar{D}-\beta \widehat{D}+\alpha L-\alpha L^{2}-\alpha \bar{E}-\alpha \widehat{E}\right)\right] x \\
& =\left(D_{\alpha \beta}+D_{1}+L_{1}-L_{\alpha \beta}\right)^{-1} \\
& \times\left[\alpha \bar{F}+\lambda\left(\alpha \bar{D}-\alpha L+\alpha L^{2}+\alpha \bar{E}\right)\right. \\
& \left.-\beta U+\beta U^{2}+\beta \widehat{F}+\lambda(\beta \widehat{D}+\beta \widehat{E})\right] x
\end{aligned}
$$

$$
\begin{gathered}
=(\lambda-1)\left(D_{\alpha \beta}+D_{1}+L_{1}-L_{\alpha \beta}\right)^{-1} \\
\times\left[\alpha L\left(D_{1}+L_{1}\right)+\alpha(\bar{D}+\bar{E})\right. \\
\left.\quad+\beta U\left(D_{1}+L_{1}\right)+\beta(U-\widehat{F})\right] x \\
=(\lambda-1)\left(D_{\alpha \beta}+D_{1}+L_{1}-L_{\alpha \beta}\right)^{-1} \\
\quad \times\left[(\alpha L+\beta U)\left(D_{1}+L_{1}\right)\right. \\
\quad+\alpha(\bar{D}+\bar{E})+\beta(U-\widehat{F})] x .
\end{gathered}
$$

If $a_{i j}+\sum_{k=i+1}^{n} a_{i k} a_{k j} \leq 0$, then by the proof of Theorem 10, we have $U-\widehat{F} \geq 0$.

Therefore, one has the following.

(1) If $\lambda \geq 1$, then $\widetilde{T} x-\lambda x \geq 0$ but not equal to 0 . Therefore, $\widetilde{T} x \geq \lambda x$. By Lemma 5, we get $\rho(\widetilde{T}) \geq \lambda=\rho(T)$.

(2) If $\lambda<1$, then $\widetilde{T} x-\lambda x \leq 0$ but not equal to 0 . Therefore, $\widetilde{T} x \leq \lambda x$. By Lemma 5, we get $\rho(\widetilde{T}) \leq \lambda=\rho(T)$.

Remark. If we choose $\alpha=0$ in Theorem 11, we have a similar result which is showed by the following corollary.

Corollary 12. Let A be a nonsingular Z-matrix. Assume that $D_{1} \geq 0,0 \leq L_{1} \leq L_{\beta}, \beta \in[0,1]$, and $\widehat{T}$ and $T$ are the iterative matrices given by (15) and (8), respectively. Consider the following.

(i) If $\rho(T)<1$, then

$$
\rho(\widehat{T})<\rho(T)<1 .
$$

(ii) Let A be irreducible. Assume that

$$
1-\beta \sum_{k=i+1}^{n} a_{i k} a_{k j}>0, \quad a_{i j}+\sum_{k=i+1}^{n} a_{i k} a_{k j} \leq 0 ;
$$

then, one has

(1) $\rho(\widehat{T}) \geq \rho(T)$ if $\rho(T) \geq 1$,

(2) $\rho(\widehat{T}) \leq \rho(T)$ if $\rho(T)<1$.

Now, one will provide some results to show the relations among $\rho(\widetilde{T}), \rho(\bar{T})$, and $\rho(\widehat{T})$.

Theorem 13. Let $A=\left(a_{i j}\right) \in R^{n \times n}$ be a nonsingular Z-matrix. Let $\widetilde{T}$ and $\bar{T}$ be iterative matrices given by (13) and (14), respectively. Assume that $\alpha, \beta \in[0,1], D_{1} \geq 0,0 \leq L_{1} \leq L_{\alpha \beta}$. If $1-\alpha \sum_{l=1}^{i-1} a_{i l} a_{l j}-\beta \sum_{k=i+1}^{n} a_{i k} a_{k j}>0$ and $a_{i j}+\sum_{k=i+1}^{n} a_{i k} a_{k j} \leq 0$, then

(1) $\rho(\widetilde{T}) \geq \rho(\bar{T})$ if $\rho(T) \geq 1$;

(2) $\rho(\widetilde{T}) \leq \rho(\bar{T})$ if $\rho(T)<1$. 
Proof. Since $D_{\alpha \beta}+D_{1}+L_{1}-L_{\alpha \beta}$ and $D_{\alpha}+D_{1}+L_{1}-L_{\alpha}$ are two lower triangular $L$-matrices with $D_{\alpha \beta}+D_{1}+L_{1}-L_{\alpha \beta} \leq$ $D_{\alpha}+D_{1}+L_{1}-L_{\alpha}$, by Lemma 9 , we have

$$
\left(D_{\alpha \beta}+D_{1}+L_{1}-L_{\alpha \beta}\right)^{-1} \geq\left(D_{\alpha}+D_{1}+L_{1}-L_{\alpha}\right)^{-1} \geq 0 \text {. }
$$

By the proof of Theorems 10 and 11, we consider

$$
\begin{aligned}
\widetilde{T} x-\bar{T} x= & \widetilde{T} x-\lambda x-(\bar{T} x-\lambda x) \\
= & (\lambda-1)\left(D_{\alpha \beta}+D_{1}+L_{1}-L_{\alpha \beta}\right)^{-1} \\
& \times\left[(\alpha L+\beta U)\left(D_{1}+L_{1}\right)+\alpha \bar{D}\right. \\
& \quad+\alpha \bar{E}+\beta(U-\widehat{F})] x \\
& -\left(D_{\alpha}+D_{1}+L_{1}-L_{\alpha}\right)^{-1} \\
& \times\left[\alpha L\left(D_{1}+L_{1}\right)+\alpha \bar{D}+\alpha \bar{E}\right] x \\
\geq & (\lambda-1)\left(D_{\alpha \beta}+D_{1}+L_{1}-L_{\alpha \beta}\right)^{-1} \\
& \times\left(\beta U\left(D_{1}+L_{1}\right)+\beta(U-\widehat{F})\right) x .
\end{aligned}
$$

In view of the proof of Theorem 11 , we have $\beta U\left(D_{1}+L_{1}\right)+$ $\beta(U-\widehat{F}) \geq 0$.

Therefore, one has the following.

(1) If $\lambda \geq 1$, the right-hand side of the above inequality is more than zero. By Lemma $8, \rho(\widetilde{T}) \geq \rho(\bar{T})$.

(2) If $\lambda<1$, the right-hand side of the above inequality is more than zero. By Lemma $8, \rho(\widetilde{T}) \leq \rho(\bar{T})$.

Theorem 14. Let $A=\left(a_{i j}\right) \in R^{n \times n}$ be a nonsingular Z-matrix. Let $\widetilde{T}$ and $\widehat{T}$ be iterative matrices given by (13) and (15), respectively. Assume that $\alpha, \beta \in[0,1], D_{1} \geq 0,0 \leq L_{1} \leq L_{\alpha \beta}$. If $1-\alpha \sum_{l=1}^{i-1} a_{i l} a_{l j}-\beta \sum_{k=i+1}^{n} a_{i k} a_{k j}>0$ and $a_{i j}+\sum_{k=i+1}^{n} a_{i k} a_{k j} \leq 0$, then

(1) $\rho(\widetilde{T}) \geq \rho(\widehat{T})$ if $\rho(T) \geq 1$,

(2) $\rho(\widetilde{T}) \leq \rho(\widehat{T})$ if $\rho(T)<1$.

Proof. Since $D_{\alpha \beta}+D_{1}+L_{1}-L_{\alpha \beta}$ and $D_{\beta}+D_{1}+L_{1}-L_{\beta}$ are two lower triangular $L$-matrices with $D_{\alpha \beta}+D_{1}+L_{1}-L_{\alpha \beta} \leq$ $D_{\beta}+D_{1}+L_{1}-L_{\beta}$, by Lemma 9 , we have

$$
\left(D_{\alpha \beta}+D_{1}+L_{1}-L_{\alpha \beta}\right)^{-1} \geq\left(D_{\beta}+D_{1}+L_{1}-L_{\beta}\right)^{-1} \geq 0 .
$$

By the proof of Corollary 12 and Theorem 11, we consider

$$
\begin{aligned}
\widetilde{T} x-\widehat{T} x= & \widetilde{T} x-\lambda x-(\widehat{T} x-\lambda x) \\
= & (\lambda-1)\left(D_{\alpha \beta}+D_{1}+L_{1}-L_{\alpha \beta}\right)^{-1} \\
& \times\left[(\alpha L+\beta U)\left(D_{1}+L_{1}\right)+\alpha \bar{D}\right. \\
& \quad+\alpha \bar{E}+\beta(U-\widehat{F})] x \\
& -\left(D_{\alpha}+D_{1}+L_{1}-L_{\alpha}\right)^{-1} \\
& \times\left[\beta U\left(D_{1}+L_{1}\right)+\beta(U-\widehat{F})\right] x \\
\geq & (\lambda-1)\left(D_{\alpha \beta}+D_{1}+L_{1}-L_{\alpha \beta}\right)^{-1} \\
& \times\left(\alpha L\left(D_{1}+L_{1}\right)+\alpha \bar{D}+\alpha \bar{E}\right) x .
\end{aligned}
$$

Since $\alpha L\left(D_{1}+L_{1}\right)+\alpha \bar{D}+\alpha \bar{E} \geq 0$, we get the following.

(1) If $\lambda \geq 1$, the right-hand side of the above inequality is more than zero. By Lemma $8, \rho(\widetilde{T}) \geq \rho(\widehat{T})$.

(2) If $\lambda<1$, the right-hand side of the above inequality is more than zero. By Lemma $8, \rho(\widetilde{T}) \leq \rho(\widehat{T})$.

Remark. The results (theorems and corollaries) in Section 3 are in some sense the generalized Stein-Rosenberg-type theorems like those in the papers [10-13]. The results (theorems and corollaries) in Section 3 are the comparisons of spectral radius of iterative matrices between the mixed-type splitting method and the preconditioned mixed-type splitting method, while the results in the papers [10-13] are the comparisons of spectral radius of iterative matrices between the parallel decomposition-type relaxation method and its special case.

\section{Numerical Example}

Consider the following equation:

$$
-\Delta u+\frac{\partial u}{\partial x}+\frac{\partial u}{\partial y}=f,
$$

in the unit square $\Omega$ with Dirichlet boundary conditions.

If we apply the central difference scheme on a uniform grid with $N \times N$ interior nodes $\left(N^{2}=n\right)$ to the discretization of the above equation, we can get a system of linear equations with the coefficient matrix

$$
A=I \otimes P+Q \otimes I
$$

where $\otimes$ denotes the Kronecker product,

$$
\begin{aligned}
& P=\operatorname{tridiag}\left(-\frac{2+h}{8}, 1,-\frac{2-h}{8}\right), \\
& Q=\operatorname{tridiag}\left(-\frac{1+h}{4}, 1,-\frac{1-h}{4}\right)
\end{aligned}
$$

are $N \times N$ tridiagonal matrices, and the step size is $h=1 / N$. 
TABLE 1

\begin{tabular}{lcccc}
\hline$\alpha(\beta)$ & $\rho(T)$ & $\rho(\bar{T})$ & $\rho(\widehat{T})$ & $\rho(\widetilde{T})$ \\
\hline 0 & 0.563691 & 0.563691 & 0.563691 & 0.563691 \\
0.05 & 0.563691 & 0.554915 & 0.554027 & 0.545537 \\
0.1 & 0.563691 & 0.545958 & 0.544027 & 0.527441 \\
0.15 & 0.563691 & 0.536827 & 0.533673 & 0.509401 \\
0.2 & 0.563691 & 0.527531 & 0.522949 & 0.491418 \\
0.25 & 0.563691 & 0.518081 & 0.511842 & 0.473488 \\
0.3 & 0.563691 & 0.508493 & 0.500331 & 0.455608 \\
0.35 & 0.563691 & 0.498782 & 0.488412 & 0.437775 \\
0.4 & 0.563691 & 0.488967 & 0.476073 & 0.419983 \\
0.45 & 0.563691 & 0.479065 & 0.463309 & 0.402227 \\
0.5 & 0.563691 & 0.469097 & 0.450121 & 0.384541 \\
\hline
\end{tabular}

We choose $N=5$; then $A \in R^{25 \times 25}$.

If we choose

(1) $D_{1}=0.45 D_{\alpha}, L_{1}=0.4 L_{\alpha}, \alpha \in[0,0.5]$,

(2) $D_{1}=0.45 D_{\beta}, L_{1}=0.4 L_{\beta}, \beta \in[0,0.5]$,

(3) $D_{1}=0.45 D_{\alpha \beta}, L_{1}=0.4 L_{\alpha \beta}, \alpha=\beta \in[0,0.5]$, then we can obtain the following results by Theorems 10-14.

Table 1 shows that that the rate of convergence of the preconditioned mixed-type splitting method is faster than that of the mixed-type splitting method. And it shows that the rate of convergence of the preconditioned mixed-type splitting method with $P_{\alpha \beta}$ is faster than that of the preconditioned mixed-type splitting method with $P_{\alpha}$ or $P_{\beta}$.

\section{Acknowledgments}

This work was supported by the National Natural Science Foundation of China (Grant no. 11001144) and the Natural Science Foundation of Shandong Province of China (ZR2012AL09).

\section{References}

[1] G. H. Cheng, T. Z. Huang, and S. Q. Shen, "Note to the mixedtype splitting iterative method for Z-matrices linear systems," Journal of Computational and Applied Mathematics, vol. 220, no. 1-2, pp. 1-7, 2008.

[2] D. M. Young, Iterative Solution of Large Linear Systems, Academic Press, New York, NY, USA, 1971.

[3] H. Schneider, "Theorems on M-splittings of a singular Mmatrix which depend on graph structure," Linear Algebra and Its Applications C, vol. 58, pp. 407-424, 1984.

[4] R. S. Varga, Matrix Iterative Analysis, Prentice-Hall, Englewood Cliffs, NJ, USA, 1962.

[5] W. Li and W. Sun, "Modified Gauss-Seidel type methods and Jacobi type methods for Z-matrices," Linear Algebra and Its Applications, vol. 317, no. 1-3, pp. 227-240, 2000.

[6] A. Berman and R. J. Plemmons, Nonnegative Matrices in the Mathematical Sciences, Academic Press, New York, NY, USA, 1979.

[7] A. Berman and R. J. Plemmons, Nonnegative Matrices in the Mathematical Sciences, SIAM, Philadelphia, PA, USA, 1994.
[8] O. Axelsson, Iterative Solution Methods, Cambridge University Press, Cambridge, UK, 1994.

[9] T. Z. Huang, G. H. Cheng, and X. Y. Cheng, "Modified SORtype iterative method for Z-matrices," Applied Mathematics and Computation, vol. 175, no. 1, pp. 258-268, 2006.

[10] Z.-Z. Bai, "On the comparisons of the multisplitting unsymmetric aor methods for M-matrices," Calcolo, vol. 32, no. 3-4, pp. 207-220, 1995.

[11] Z.-Z. Bai, "The generalized Stein-Rosenberg type theorem for the PDAOR-method," Bulletin of the Institute of Mathematics Academia Sinica, vol. 19, pp. 329-335, 1997 (Chinese).

[12] Z.-Z. Bai, "A class of parallel decomposition-type relaxation methods for large sparse systems of linear equations," Linear Algebra and Its Applications, vol. 282, no. 1-3, pp. 1-24, 1998.

[13] Z.-Z. Bai and R. Nabben, "Some properties of the block matrices in the parallel decomposition-type relaxation methods," Applied Numerical Mathematics, vol. 29, no. 2, pp. 167-170, 1999. 


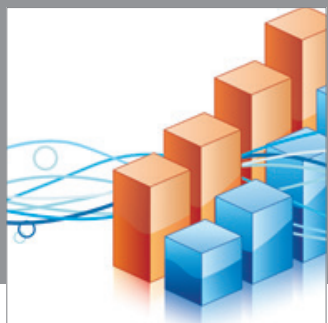

Advances in

Operations Research

mansans

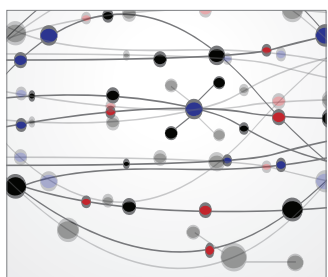

The Scientific World Journal
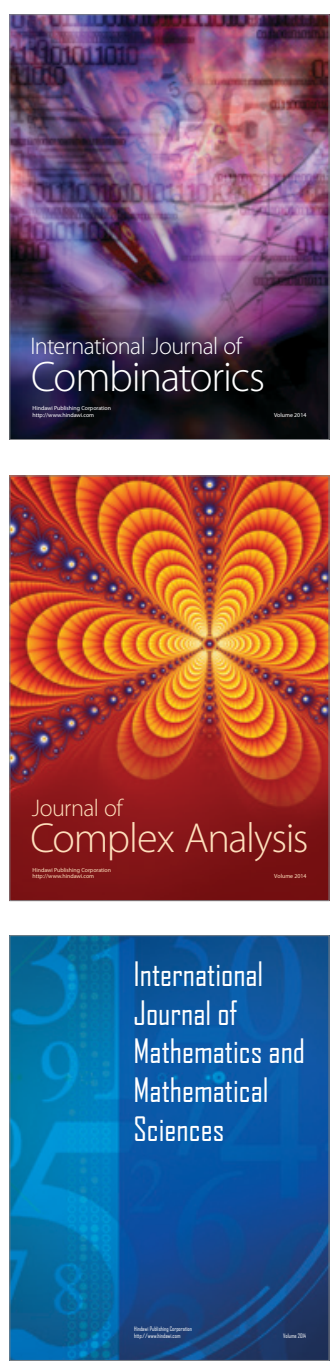
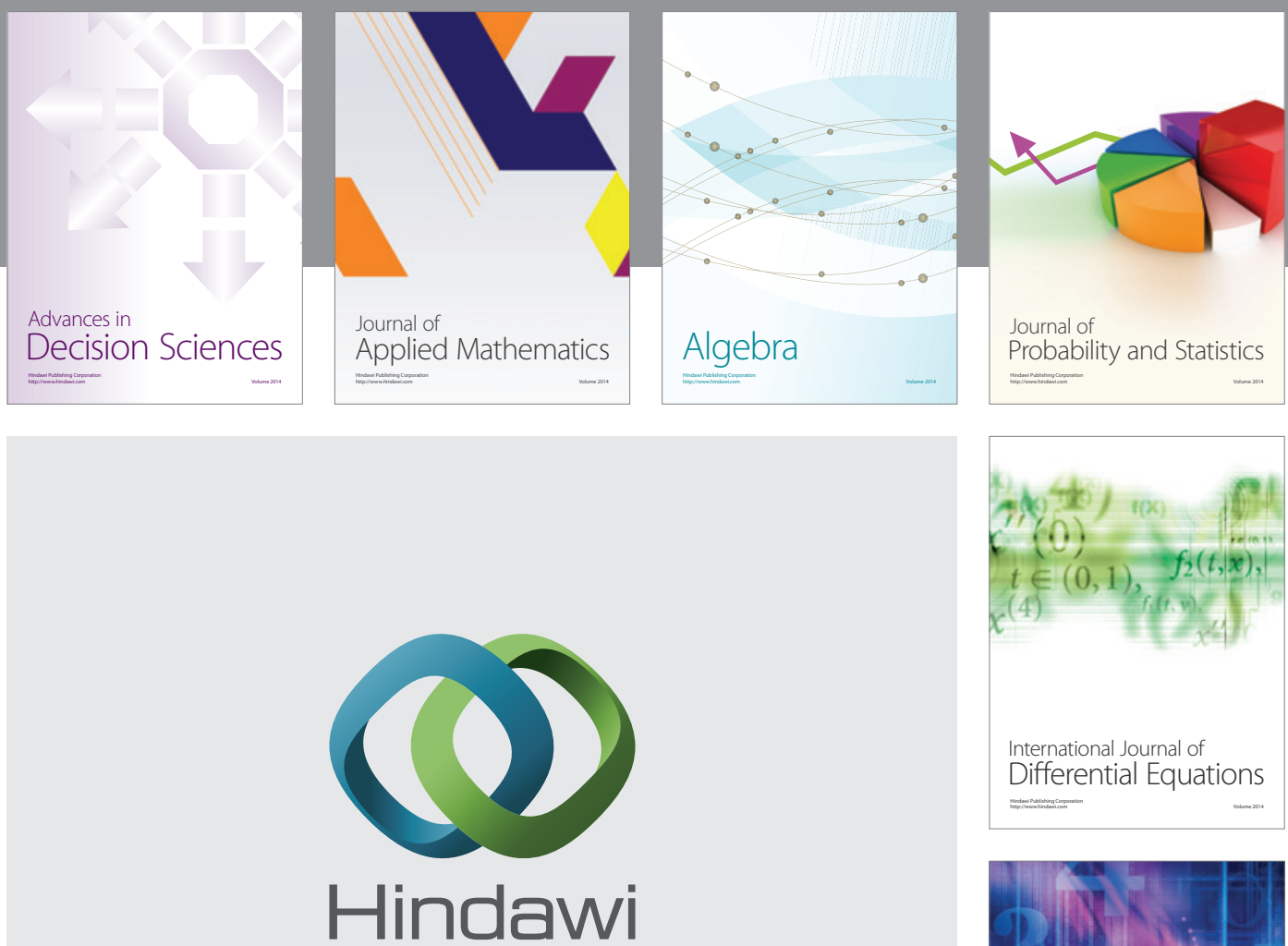

Submit your manuscripts at http://www.hindawi.com
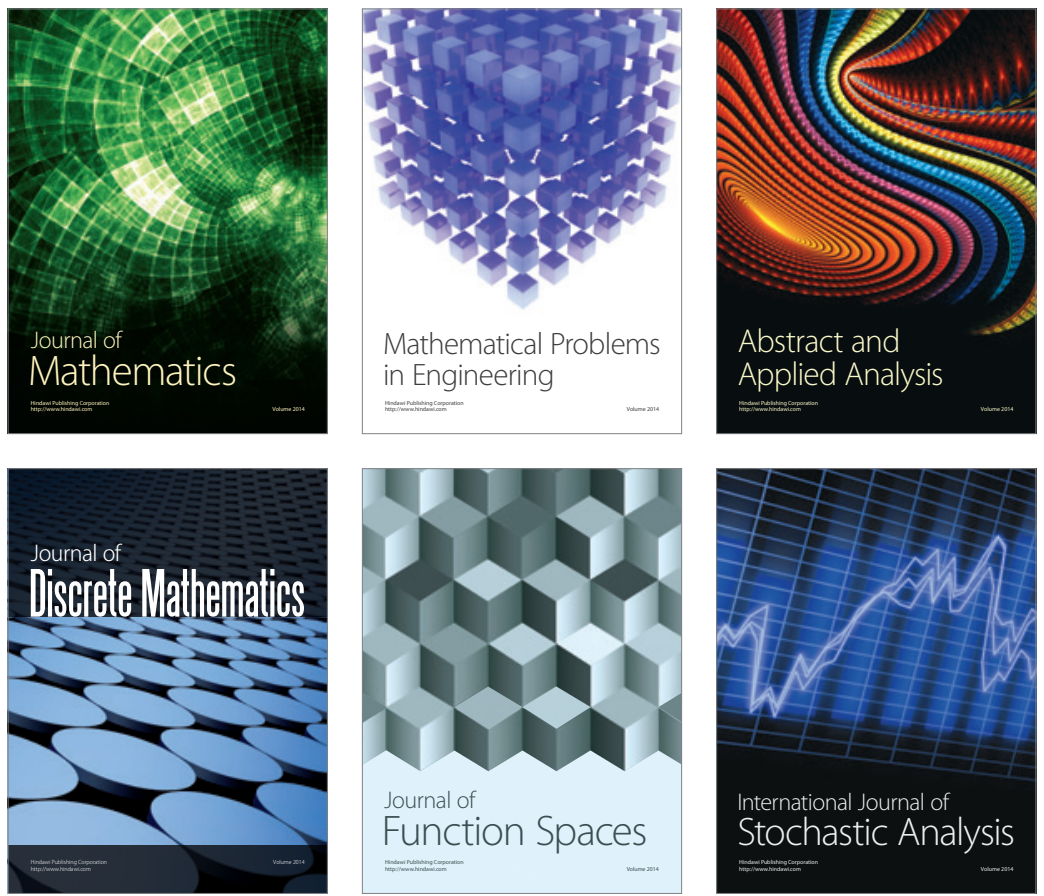

Journal of

Function Spaces

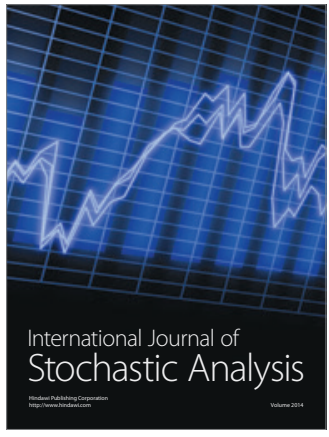

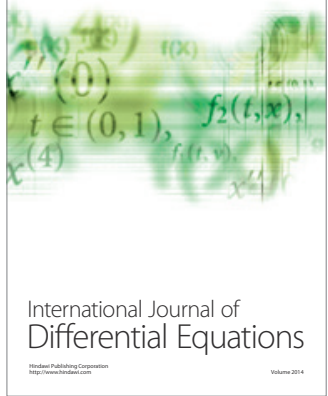
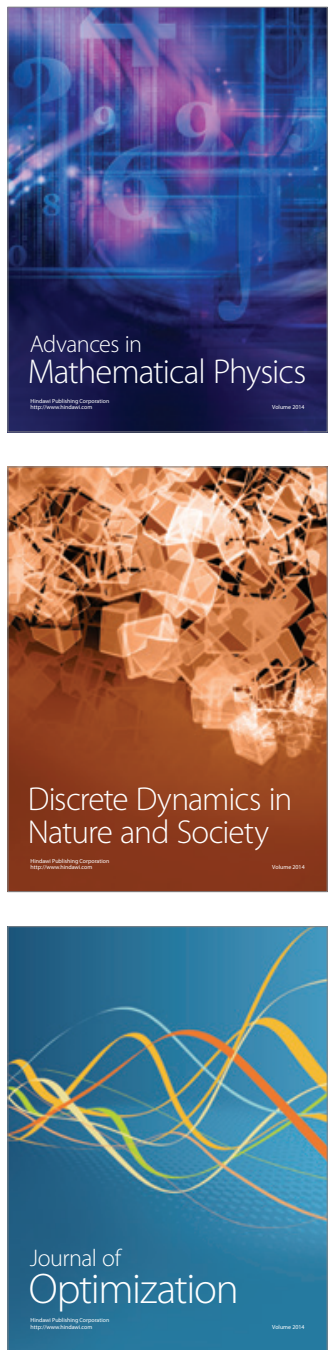